H. Ono

Nagoya Math. J.

Vol. 38 (1970), 63-69

\title{
SOME REMARKS ON EVALUATIONS OF THE PRIMITIVE LOGIG
}

\section{HIROAKIRA ONO}

\section{Dedicated to Prof. K. Ono on his 60th birthday}

In [4], K. Ono introduced the notion of evaluations of the primitive logic $\boldsymbol{L O}$ and proved that any semi-evaluation $\boldsymbol{E}$ is an evaluation of $\boldsymbol{L O}$ if $\boldsymbol{E}$ satisfies the following conditions:

$$
\begin{aligned}
& (E 1) \quad p^{*} \longrightarrow 0=0, \\
& (E 2) \quad p^{*} \longrightarrow p^{*}=0, \\
& (E 3) \quad 0 \longrightarrow p^{*}=p^{*}, \\
& (E 4) \quad p^{*} \longrightarrow\left(p^{*} \longrightarrow q^{*}\right)=p^{*} \longrightarrow q^{*}, \\
& (E 5) \quad p^{*} \longrightarrow\left(q^{*} \longrightarrow r^{*}\right)=q^{*} \longrightarrow\left(p^{*} \longrightarrow r^{*}\right), \\
& (E 6) \quad p^{*} \longrightarrow q^{*}=0 \text { implies } \\
& \quad\left(r^{*} \longrightarrow p^{*}\right) \longrightarrow\left(r^{*} \longrightarrow q^{*}\right)=0, \\
& (E 7) \quad(x) p^{*}(x) \longrightarrow p^{*}(t)=0 \text { for any } t, \text { and } \\
& (E 8) \text { if } u^{*} \longrightarrow p^{*}(t)=0 \text { for any } t \text {, then }
\end{aligned}
$$

$$
u^{*} \longrightarrow(x) p^{*}(x)=0 .
$$

That is, if $\boldsymbol{A}$ is provable in $\boldsymbol{L O}$, then for any semi-evaluation $\boldsymbol{E}$ satisfying the above conditions, $\boldsymbol{E}(\boldsymbol{A})=0$ holds identically. In this paper, we will show that in \$1, the condition $(E 8)$ is so weak that we can not prove the above result and hence (E8) must be replaced by the following condition $\left(E 8^{*}\right)$;

$$
\begin{aligned}
\left(E 8^{*}\right) \text { if } u^{*} \longrightarrow\left(v^{*} \longrightarrow p^{*}(t)\right)=0 \text { for any } t, \text { then } \\
\qquad u^{*} \longrightarrow\left(v^{*} \longrightarrow(x) p^{*}(x)\right)=0,
\end{aligned}
$$

and that in $\$ 2$, converse of his result can be proved if (E8) is replaced by (E8*).

\$1. We first define a model $\boldsymbol{D}$ of $\boldsymbol{L O}$, after the definition of evaluations. Let $\boldsymbol{D}$ be an algebraic structure $\langle D ; 0, \longrightarrow, V\rangle$, where $D$ and $V$ are sets,

Received March 29, 1969 
$0 \in D$ and $\longrightarrow$ is a function from $D^{2}$ to $D$. If $\boldsymbol{D}$ satisfies the following conditions, we say that $\boldsymbol{D}$ is a model of $\boldsymbol{L O}$.

For each $p, q$ and $r \in D$,

(D1) $\quad p \longrightarrow 0=0$,

(D2) $p \longrightarrow p=0$,

(D3) $0 \longrightarrow p=p$,

(D4) $p \longrightarrow(p \longrightarrow q)=p \longrightarrow q$,

(D5) $p \longrightarrow(q \longrightarrow r)=q \longrightarrow(p \longrightarrow r)$,

(D6) if $p \longrightarrow q=0$ then $(r \longrightarrow p) \longrightarrow(r \longrightarrow q)=0$.

Suppose that $a_{t} \in D$ for any $t \in V$. Then there exists an element $\operatorname{top}\left[a_{t} \mid t \in V\right]$ in $D$ which satisfies the following conditions ${ }^{1}$.

(D7) $\quad t o p\left[a_{t} \mid t \in V\right] \longrightarrow a_{t}=0$ for any $t \in V$,

(D8) if $p \longrightarrow\left(q \longrightarrow a_{t}\right)=0$ for any $t \in V$,

$$
\text { then } p \longrightarrow\left(q \longrightarrow \operatorname{top}\left[a_{t} \mid t \in V\right]=0\right. \text {. }
$$

Let $\boldsymbol{D}$ be a model of $\boldsymbol{L} \boldsymbol{O}$ and $\varphi$ be a mapping. from the class of primitive symbols of $\boldsymbol{L O}$ such that $\varphi(v) \in V$ for each variable or constant $v$ and if $p$ is an $n$-ary function symbol then $\varphi(p)$ is a mapping from $V^{n}$ to $D$. Then we say that this $\varphi$ is an assignment over $\boldsymbol{D}$. Although $\varphi$ is defined only for primitive symbols, we can extend the domain of the mapping $\varphi$ to the class of formulas in a natural way. That is,

$\varphi\left(p\left(t_{1}, \cdots, t_{m}\right)\right)=\varphi(p)\left[\varphi\left(t_{1}\right), \cdots, \varphi\left(t_{m}\right)\right]$ where right side of the equality means the value of $\varphi(p)$ for the $m$-tuple $\left[\varphi\left(t_{1}\right), \cdots, \varphi\left(t_{m}\right)\right]$,

$$
\begin{aligned}
& \varphi(P \longrightarrow Q)=\varphi(P) \longrightarrow \varphi(Q) \text { and } \\
& \varphi((x) P(x))=\operatorname{top}[\varphi(P(x)) \mid x \in V] .
\end{aligned}
$$

$\boldsymbol{A}$ is valid in a model $\boldsymbol{D}$ if $\varphi(\boldsymbol{A})=0$ for any assignment $\varphi$ over $\boldsymbol{D}$. Now we can prove the following lemma easily.

LEMma 1. For any semi-evaluation $\boldsymbol{E}$ satisfying from $(E 1)$ to $(E 7)$ and $\left(E 8^{*}\right)$, there exists a model of $\boldsymbol{L O}$ and an assignment $\varphi$ over $\boldsymbol{D}$ such that $\varphi(p)=\boldsymbol{E}(p)$ for any function symbol $p$. Conversely, for each model $\boldsymbol{D}$ of $\boldsymbol{L O}$ and each assignment $\varphi$ over $\boldsymbol{D}$, there exists a semi-evaluation $\boldsymbol{E}$ such that $\varphi(p)=\boldsymbol{E}(p)$.

1) The word "top" is due to Henkin. See Henkin [2]. 
Notice that we can also prove Lemma 1 when we replace $\left(E 8^{*}\right)$ by $(E 8)$ and $(D 8)$ by $\left(D 8^{\prime}\right)$ defined as follows, in Lemma 1.

$\left(D 8^{\prime}\right) \quad$ If $p \longrightarrow a_{t}=0$ for any $t \in V$, then

$$
p \longrightarrow t o p\left[a_{t} \mid t \in V\right]=0 .
$$

In the proof of Theorem 2 in [4], K. Ono asserted that it can be proved by using (E8) that

$$
\begin{aligned}
& \text { if } p_{1} \longrightarrow\left(p_{2} \longrightarrow\left(\cdots\left(p_{n} \longrightarrow q(t)\right) \cdots\right)\right)=0 \text { for any } t \text {, } \\
& \text { then } p_{1} \longrightarrow\left(p_{2} \longrightarrow\left(\cdots\left(p_{n} \longrightarrow(x) q(x)\right) \cdots\right)\right)=0
\end{aligned}
$$

However we can prove this is not the case, by constructing a structure which satisfies the conditions from $(D 1)$ to $(D 7)$ and $\left(D 8^{\prime}\right)$, but in which (1) does not hold. We construct a structure $\boldsymbol{B}=\langle B, 0, \longrightarrow, V\rangle$ as follows. $B$ is a Boolean lattice whose cardinality is 4 (see Fig. 1) and $V=\left\{v_{1}, v_{2}\right\} .0$ is a minimal element of $B$. Define the value of $p \longrightarrow q$ and of $t o p\left[p_{t} \mid t \in V\right]$ as follows.

$$
p \longrightarrow q= \begin{cases}0 & \text { if } p \geqq q \\ q & \text { otherwise }\end{cases}
$$

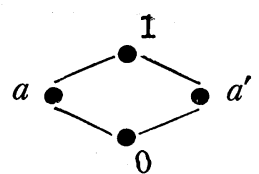

Figure 1

We can see easily that $\boldsymbol{B}$ satisfies the conditions from $(D 1)$ to $(D 7)$ and $\left(D 8^{\prime}\right)$. Now suppose that an assignment $\varphi$ over $\boldsymbol{B}$ is defined as follows.

$$
\varphi\left(x_{1}\right)=v_{1}, \varphi\left(x_{i}\right)=v_{2} \text { if } x_{i} \neq x_{1}, \varphi\left(p_{1}\right)=a, \varphi\left(p_{2}\right)=a^{\prime} \text {, and } \varphi(q)=f \text { where }
$$
$f\left(v_{1}\right)=a$ and $f\left(v_{2}\right)=a^{\prime}$. Then we can prove the following lemma.

Lemma 2. $\varphi\left(p_{1} \longrightarrow\left(p_{2} \longrightarrow q(x)\right)\right)=0$ for any $x$, but $\varphi\left(p_{1} \longrightarrow\left(p_{2} \longrightarrow\right.\right.$ $(x) q(x))) \neq 0$. Therefore (1) can not be deduced from the conditions from (E1) to $(E 8)$.

Now we will show that the condition $\left(E 8^{*}\right)$ is sufficient to prove (1). 
Lemma 3. (1) is deducible from the conditions from (E1) to (E7) and (E8*).

Proof. We use $\left[p_{1}, \cdots, p_{n}\right]-* \rightarrow q$ as an abbreviation for $p_{1} \longrightarrow\left(p_{2} \longrightarrow\right.$ $\left.\left(\cdots\left(p_{n} \longrightarrow q\right) \cdots\right)\right)$. More precisely, we define $\left[p_{1}, \cdots, p_{n}\right]-* \rightarrow q$ inductively as follows. For $n=0,\left(\left[p_{1}, \cdots, p_{n}\right]-* \rightarrow q\right)=q$ and for $n>0, \quad\left(\left[p_{1}, \cdots, p_{n}\right]\right.$ $-* \rightarrow q)=\left(p_{1} \longrightarrow\left(\left[p_{2}, \cdots, p_{n}\right]-* \rightarrow q\right)\right)$.

We shall first show that

$$
(x)\left(\left[p_{1}, \cdots, p_{m}\right]-_{* \rightarrow q}(x)\right) \longrightarrow\left(\left[p_{1}, \cdots, p_{m}\right]-* \rightarrow(x) q(x)\right)=0
$$

by using induction on $m$.

For $m=0$, the left side of (2) is equal to $(x) q(x) \longrightarrow(x) q(x)$. Then (2) is deducible from (E2).

Suppose that $m>0$. By (E7),

$$
\begin{gathered}
(x)\left(p_{1} \longrightarrow\left(\left[p_{2}, \cdots, p_{m}\right]-* \rightarrow q(x)\right)\right) \longrightarrow\left(p_{1} \longrightarrow\left(\left[p_{2}, \cdots, p_{m}\right] \rightarrow * \rightarrow q(t)\right)\right)=0 \\
\text { for any } t .
\end{gathered}
$$

Taking $(x)\left(p_{1} \longrightarrow\left(\left[p_{2}, \cdots, p_{m}\right]-* \rightarrow q(x)\right)\right)$ as $u^{*}, p_{1}$ as $v^{*}$ and $\left[p_{2}, \cdots, p_{m}\right]$ -* $\rightarrow q(t)$ as $p^{*}(t)$, and using $\left(E 8^{*}\right)$, we have

$$
(x)\left(p_{1} \longrightarrow\left(\left[p_{2}, \cdots, p_{m}\right]-* \rightarrow q(x)\right)\right) \longrightarrow\left(p_{1} \longrightarrow(x)\left(\left[p_{2}, \cdots, p_{m}\right]-* \rightarrow q(x)\right)\right)=0
$$

By induction hypothesis,

$$
(x)\left(\left[p_{2}, \cdots, p_{m}\right]-* \rightarrow q(x)\right) \longrightarrow\left(\left[p_{2}, \cdots, p_{m}\right]-* \rightarrow(x) q(x)\right)=0 .
$$

From (E3), (E6) and (3), we get

$$
(x)\left(\left[p_{1}, \cdots, p_{m}\right] \longrightarrow * \rightarrow q(x)\right) \longrightarrow\left(\left[p_{1}, \cdots, p_{m}\right]-* \rightarrow(x) q(x)\right)=0 .
$$

Now, since $\left[p_{1}, \cdots, p_{n}\right]-* \rightarrow q(t)=0$ for any $t, p_{1} \longrightarrow(x)\left(\left[p_{2}, \cdots, p_{n}\right] \rightarrow * \rightarrow\right.$ $q(x))=0$ from $\left(E 8^{*}\right)$. For, $\left(E 8^{*}\right)$ implies (E8). Using (2) and (E6),

$$
\begin{aligned}
& \left(p_{1} \longrightarrow(x)\left(\left[p_{2}, \cdots, p_{n}\right] \rightarrow * \rightarrow q(x)\right)\right) \longrightarrow\left(\left[p_{1}, \cdots, p_{n}\right]-* \rightarrow(x) q(x)\right) \\
& =0 \longrightarrow\left(\left[p_{1}, \cdots, p_{n}\right] \longrightarrow * \rightarrow(x) q(x)\right)=0 .
\end{aligned}
$$

Thus we have $\left[p_{1}, \cdots, p_{n}\right]-* \rightarrow(x) q(x)=0$.

Now, we get the following theorem after K. Ono's proof of Theorem 2 in [4].

Theorem 1. If $\boldsymbol{A}$ is provable in LO, then $\boldsymbol{A}$ is valid in any model of LO. 
§2. We will prove the converse of Theorem 1. Suppose that $\boldsymbol{A}$ is valid in any model of $\boldsymbol{L O}$. Since any Brouwerian algebra satisfies the conditions from $(D 1)$ to $(D 8), \boldsymbol{A}$ is valid in any Brouwerian algebra ${ }^{2)}$. Rasiowa proved in [6] that for any formula $\boldsymbol{B}$ in the intuitionistic logic $\boldsymbol{L} \boldsymbol{J}$, if $\boldsymbol{B}$ is valid in any Brouwerian algebra then $\boldsymbol{B}$ is provable in $\boldsymbol{L} \boldsymbol{J}$. Thus $\boldsymbol{A}$ is provable in $\boldsymbol{L J}$. Moreover $\boldsymbol{A}$ is a formula of $\boldsymbol{L O}$, and hence $\boldsymbol{A}$ is provable in $\boldsymbol{L O}$ by using Gentzen's Hauptsatz ${ }^{3}$.

Theorem 2. If $\boldsymbol{A}$ is valid in any model of LO, then $\boldsymbol{A}$ is provable in $\mathrm{LO}^{4}$.

$\mathrm{K}$. Ono gave me a preprint of his new paper [5], in which he defines the set-theoretical (or topological) interpretation of $\boldsymbol{L O}$. Also in this paper, the condition $(E 8)$ should be replaced by $\left(E 8^{*}\right)$. We will discuss the matter in $\S 3$.

§3. We must revise the conditions which make any pair of topologies ( $\{\boldsymbol{T}\},[\boldsymbol{T}])$ logical as follows.

Definition. Any pair of topologies ( $\{\boldsymbol{T}\},[\boldsymbol{T}])$ is logical if and only if " $\longrightarrow$ " and "(x)" defined in [5] satisfy (E5) and (E8*) for every closed set $p$, $q, r$ and $a_{t}$ for any $t$ with respect to the topology $\{\boldsymbol{T}\}$.

Lemma 4. Suppose that $p, q$ and $r$ are closed sets with respect to $\{\boldsymbol{T}\}$. Then $\left[[r-q]^{r} \cap(r-p)\right]^{r}=0$ if and only if $[r-q] \cap(r-p)=0$.

Proof. For any set $a, a \subset[a]$. Hence if $\left[[r-q]^{r} \cap(r-p)\right]^{r}=0$, then $[r-q] \cap r \cap(r-p) \cap r=[r-q] \cap(r-p)=0$. Conversely, if $[r-q] \cap(r-p)=$ $[r-q]^{r} \cap(r-p)=0$ then $\left[[r-q]^{r} \cap(r-p)\right]^{r}=[0]^{r}=0$.

Corollary ${ }^{5}$. Any pair of topologies $(\{\boldsymbol{T}\},[\boldsymbol{T}])$ is logical if

(T5) $\quad[[r-p] \cap(r-q)]=[(r-p) \cap[r-q]]$

and

(T7) if $\left[a_{t}-q\right] \cap\left(a_{t}-p\right)=0$ for any $t$, then

$$
\left[\left\{\cup a_{x}\right\}-q\right] \cap\left(\left\{\cup a_{x}\right\}-p\right)=0
$$

2) For the definition of Brouwerian algebras, see, e.g., Rasiowa [6].

3) See, Curry [1].

4) We can prove this theorem directly, by using Henkin's method in his [2]. See Also [7].

5) Cf. Theorem 8 of [5]. In this corollary, $\cup a_{x}$ denotes the union of a class of sets $a_{t}$ where $t$ runs over a set $V$. 
holds for $p, q, r$ and $a_{t}$ with respect to $\{\boldsymbol{T}\}$.

We can prove each example of pair of topologies given in (2.1) and (2. 2) of [5] to be logical by the above corollary. In particular, the following lemma holds.

Lemma 56) Any pair ([T] $[\boldsymbol{T}]$ ) of identical topology $[\boldsymbol{T}]$ is logical (in our sense) if $[\boldsymbol{T}]$ satisfies the condition (T6).

From this, we shall prove the following theorem.

Theorem 3. If $\boldsymbol{A}$ is identically equal to 0 for any topological interpretation of $\boldsymbol{L O}$, then $\boldsymbol{A}$ is provable in $\boldsymbol{L O}$.

Proof. McKinsey and Tarski showed that for any Brouwerian algebra $\boldsymbol{B}$ there exists a topological space $\boldsymbol{X}$ such that $\boldsymbol{B}$ is isomorphic to $\mathscr{C}(\boldsymbol{X})$ where $\mathscr{C}(\boldsymbol{X})$ is the class of closed subsets of $\boldsymbol{X}^{7}$. On the other hand, in Rasiowa's proof, she used the fact that if $\boldsymbol{B}$ is valid in the Lindenbaum algebra $\boldsymbol{B}_{h}$ then $\boldsymbol{B}$ is provable in $\boldsymbol{L J}^{8)}$. Of course, $\boldsymbol{B}_{h}$ is a Brouwerian algebra. Hence there exists a topological space $\boldsymbol{X}_{0}$ such that $\boldsymbol{B}_{h}$ is isomorphic to $\mathscr{C}\left(\boldsymbol{X}_{0}\right)$. Clearly, the pair $\left(\left[\boldsymbol{T}_{0}\right],\left[\boldsymbol{T}_{0}\right]\right)$ of topology $\left[\boldsymbol{T}_{0}\right]$ whose class of all closed sets is $\mathscr{C}\left(\boldsymbol{X}_{0}\right)$ determines a topological interpretation $\boldsymbol{L}$ of $\boldsymbol{L O}$ by Lemma $5^{9)}$. It follows that $\boldsymbol{A}$ is identically equal to 0 for the interpretation $\boldsymbol{L}$ and hence $\boldsymbol{A}$ is valid in $\boldsymbol{B}_{h}$. Thus $\boldsymbol{A}$ is provable in $\boldsymbol{L} \boldsymbol{O}$.

Corollary. Following propositions are equivalent. For any formula $\boldsymbol{A}$ of $\boldsymbol{L O}$,

1).. $\boldsymbol{A}$ is provable in $\mathbf{L O}$,

2) $\boldsymbol{A}$ is valid in any model of $\boldsymbol{L O}$,

3) $\boldsymbol{A}$ is valid in the model $\boldsymbol{B}_{h}$,

4) $\boldsymbol{A}$ is identically equal to 0 for any topological interpretation of $\boldsymbol{L O}$.

Although we know certain relations hold between the logic $\boldsymbol{L O}$ and the model which satisfies from $(E 1)$ to $(E 7)$ and $\left(E 8^{*}\right)$, by the above corollary, another point of view is possible. What can be said about the relations between $\boldsymbol{L} \boldsymbol{O}$ and the formal system whose axioms are propositions

\footnotetext{
6) Cf. Theorem 12 of [5]. (T6) $[a] \cup[b]=[a \cup b$ ?.

7) See [3].

8) See [6].

9) See [5].
} 
from $(E 1)$ to $(E 7)$ and $\left(E 8^{*}\right)$ ? It seems that answers of this problem imply some meaningful results about the relations between logics.

\section{REFERENCES}

[1] H. Curry, A note on the reduction of Gentzen's calculus LJ, Bull. Amer. Math. Soc., 45 (1939) 288-293.

[2] L. Henkin, An algebraic characterization of quantifiers, Fund. Math., 37 (1950) 63-74.

[ 3 ] J.C.C. McKinsey and A. Tarski, On closed elements in closure algebras, Ann. of Math., 47 (1946) 122-162.

[4] K. Ono, On a class of truth-value evaluations of the primitive logic, Nagoya Math. J., 31 (1968) $71-80$.

[5] - On a class of set-theoretical interpretations of the primitive logic, (to appear).

[6] H. Rasiowa, Algebraic treatment of the functional calculi of Heyting and Lewis, Fund. Math., 38 (1951) 99-126.

[ 7 ] H. Rasiowa and R. Sikorski, The mathematics of metamathematics, Warszawa, 1963, Monografie Matematyczne tom 41 .

Research institute for mathematical sciences,

Kyoto University 